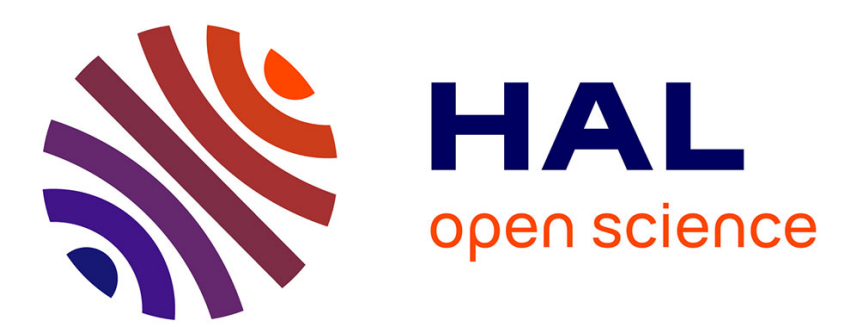

\title{
Robust Nanomanipulation Control based on Laser Beam Feedback
}

\author{
Nabil Amari, David Folio, Antoine Ferreira
}

\section{To cite this version:}

Nabil Amari, David Folio, Antoine Ferreira. Robust Nanomanipulation Control based on Laser Beam Feedback. IEEE/RSJ International Conference on Intelligent Robots and Systems (IROS'2014), Sep 2014, Chicago, IL, USA, United States. pp.6. hal-01070682

\section{HAL Id: hal-01070682 \\ https://hal.science/hal-01070682}

Submitted on 2 Oct 2014

HAL is a multi-disciplinary open access archive for the deposit and dissemination of scientific research documents, whether they are published or not. The documents may come from teaching and research institutions in France or abroad, or from public or private research centers.
L'archive ouverte pluridisciplinaire HAL, est destinée au dépôt et à la diffusion de documents scientifiques de niveau recherche, publiés ou non, émanant des établissements d'enseignement et de recherche français ou étrangers, des laboratoires publics ou privés. 


\title{
Robust Nanomanipulation Control based on Laser Beam Feedback
}

\author{
Nabil Amari, David Folio and Antoine Ferreira
}

\begin{abstract}
This paper presents a study of the control problem of a laser beam illuminating and focusing a microobject subjected to dynamic disturbances using light intensity for feedback only. The main idea is to guide and track the beam with a hybrid micro/nanomanipulator which is driven by a control signal generated by processing the beam intensity sensed by a four-quadrant photodiode. Since the pointing location of the beam depends on real-time control issues related to temperature variation, vibrations, output intensity control, and collimation of the light output, the 2-D beam location to the photodiode sensor measurement output is estimated in real-time. To overcome this problem, the Particle Filter (PF) algorithm is used to estimate the position of laser beam. The dual manipulators are controlled by combining different performance dynamics (micro and nano manipulators) in order to track a laser beam with very-high precision in a dynamics operating mode.
\end{abstract}

\section{INTRODUCTION}

Beam analysis (laser beam, or X-ray radiation) represents a great tool to improve the comprehension of physical phenomena of different types of objects at the micro or nanoscale [1][2]. A typical application is in biology where it is needed to diagnose different pathologies of cellular samples using an infrared beam. However, the main difficulty is to maintain the microsample in the focus of the laser beam with a tradeoff between robustness of the tracking and precision of the focal point (e.g., in few micrometer size spots). Usually, an atomic force microscope (AFM) nanogripper constituted by two AFM tips is used to handle and to maintain the cell under the field of focus of the laser beam[3][4][5]. For characterization purposes, the sample should be maintained in a stable way during for long periods of time with the capacity to reject the external disturbances. These perturbations are produced by the nanomanipulator platform that is subjected to mechanical microvibrations, actuator thermal drifts, photodetector noises, and brownian motion of the laser beam. Furthermore, the atmospheric turbulences are critical for the success of such AFM-based nanomanipulation tasks [6][7][8]. Classically, the task sequencing is defined by three main tasks (see Fig.1): i) detection and selection of the microobject, ii) stable nanohandling, and iii) transportation of the microobject in the focus of the laser beam. Such procedures usually involve a two-fingered AFM-based nanomanipulation system with different types of coarse and fine stages with different bandwidth dynamics, position precision, travel range (from few nanometers to hundreds micrometers) and actuation principles (magnetic and piezoelectric-stack

N. Amari, D. Folio and A. Ferreira are with the Laboratoire PRISME EA 4229; Ecole Nationale Supérieure d'Ingénieurs de Bourges, 88 boulevard Lahitolle, 18020 Bourges, France. Corresponding author: Antoine Ferreira (Email:antoine.ferreira@ensi-bourges.fr, Tel: +332 4848 4079)

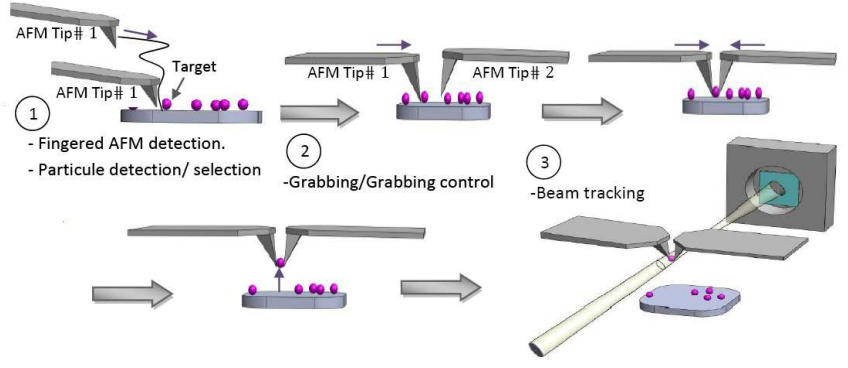

Fig. 1. High-precision position measurement based on laser beam transmission are commonly used in nanomanipulation applications.

actuated nanomanipulators)[9][10][11]. Different control architectures have been developed for dual-stage servo control schemes to deal with uncertainties (modeling errors, sensor limitations) and environment noises[12][13]. They can be classified into two categories: those based on decoupled or sequential single-input-single-output (SISO) designs, and those based on modern optimal design methodologies, such as LQG, LQG/LTR, $H_{\infty}$, and $\mu$-synthesis, in which the dual-stage controllers are obtained simultaneously. Another problem to deal with is to ensure that the microsample is maintained in the focus of the beam that possess a section area of few squared micrometers. Due to brownian motion and micromechanical vibrations perturbations, the beam tracking is a difficult problem since the microsample should be illuminated by the maximum beam intensity at the focal point (as shown in Fig.1)) during the characterization task.

We studied in this work the robust control issues of the dual AFM-based micro/nano manipulator motions to ensure the tracking of the laser beam with micro/nanometric resolution. First, robust control strategies for magnetic and piezoelectric-stack actuated nanomanipulators are implemented to deal with uncertainties (modeling errors, sensor limitations) and environment noise. Then, efficient robust algorithms are proposed to track the handling position variations due to beam exposition (electrostatic forces, Brownian motion, scattering) based on the Particle Filter (PF) algorithm. Secondly, a $H_{\infty}$ controller is designed to deal with uncertainties (modeling errors, sensor limitation, non linear effects) and environment noise. Moreover, the cooperative control scheme has to coordinate two nanogrippers mounted in real-time on a 6 degree of freedom (dof) dual-stage micro/nanomanipulator system.

The paper is divided into five sections. Section 2 describes the experimental setup composed of a two-fingered AFMbased nanomanipulation system operating under the field of 


\section{CONFIDENTIAL. Limited circulation. For review only.}

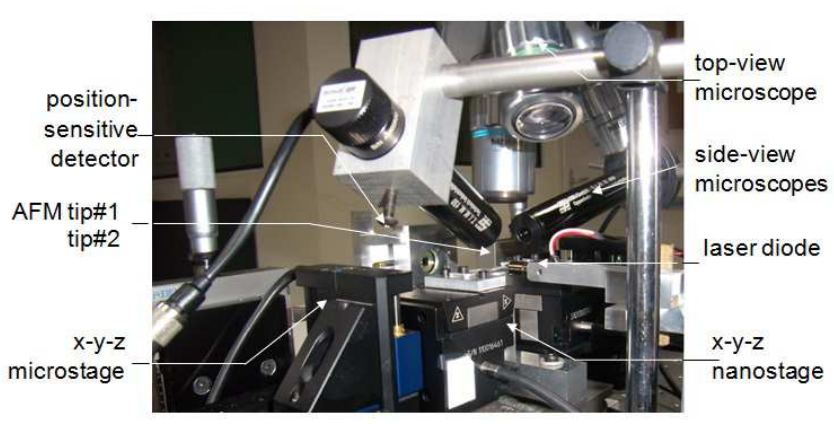

Fig. 2. Experimental setup.

focus of a laser beam emitting at infrared. Then, section 3 describes the dynamics modeling of the system. Section 4 describes the master-slave controller scheme with a decoupling structure for the laser beam tracking and nanogripper handling. Finally, section 5 presents experimental results that demonstrates the robust performances of the laser beam tracking system.

\section{EXPERIMENTAL SETUP}

The two-fingered AFM-based nanomanipulation system is illustrated in Fig.2. First, the handling system comprises two atomic force microscope (AFM) probes (Kleindiek FMT400) each mounted on two computer-controllable micro/nano manipulators facing each other. Each micro/nano manipulator is composed of 6 DoF high-precision dual-stages: a $x-y$ $z$ closed-loop nanostage (P-611.3S NanoCube from Physics Instruments) with a fine motion in the range of $120 \times$ $120 \times 120 \mu \mathrm{m}$ which is mounted on a $x-y-z$ closed-loop microstage (F-130 DC from Physics Instruments) with a coarse motion in the range of $15 \times 15 \times 15 \mathrm{~cm}$. On the side view, the laser beam is generated from a $635 \mathrm{~nm}$ laser diode (Red), and a four-quadrant position sensing device (PSD) measures the position of the image that the laser beam forms on a fixed plane. The imaging system is composed of a top-view (optical microscope - Mituyo $\times 50$ ) and side-view (digital microscope - TIMM $\times 400$ ) used for localization and guidance. Fig. 3 shows the control scheme of the two AFM probes to detect, grab, transport a microball in the focus of the laser beam, and the laser beam motion tracking (Brownian or stochastic trajectory) are processed in real-time using MATLAB $^{\odot}$ xPC software. For laser beam intensity maximum detection and beam tracking a highspeed data acquisition (DAQ) (NI 6289) card is used to register photodiode voltage output, generated when the PSD is illuminated. A multi-thread structure is developed to independently control and coordinate the two AFM tips for parallel manipulation.

\section{DYNAMICS MODELING}

This section reviews the different model dynamics of the different system components.

\section{A. Dynamics of Piezoelectric and Magnetic Actuators}

The micromanipulation process needs the cooperative control of both AFM probes in order to handle and to track, in

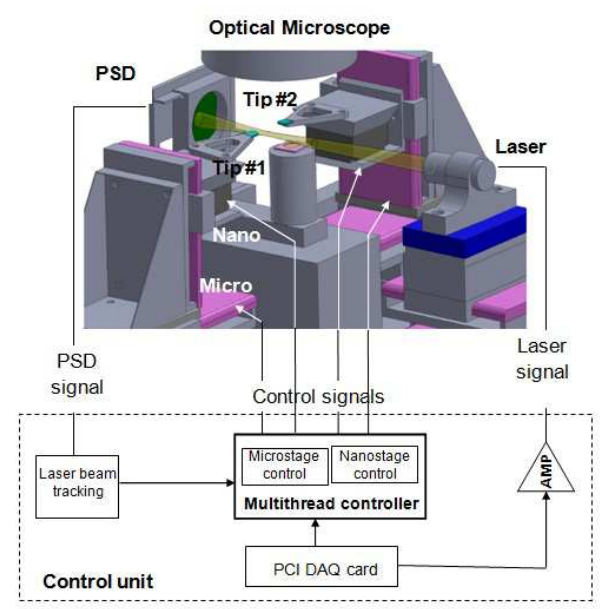

Fig. 3. Schematic diagram of the architecture of the laser beam tracking control system.

real-time, the microsample under the field of focus of the laser beam detected by the PSD. The cooperative controller sends commands to the micro/nano stages along the $x-y$ $z$ directions. As no parametric information on drivers are available, an identification phase is needed to set up dynamic modeling of dual micro/nanostages. We identified the dynamics of our dual micro/nano manipulators in $x$ - $y$-z-directions where the dynamic models of the micro and nanostages are chosen as a third-order approximation reduced order for each $x-y-z$ axis, respectively:

$$
\begin{aligned}
G_{\operatorname{micro}(x, y, z)}(z) & =\frac{b_{0}+b_{1} z^{-1}+b_{2} z^{-2}+b_{3} z^{-3}}{1+a_{1} z^{-1}+a_{2} z^{-2}+a_{3} z^{-3}} \\
G_{\operatorname{mano}(x, y, z)}(z) & =\frac{b_{1} z^{-1}+b_{2} z^{-2}+b_{3} z^{-3}}{1+a_{1} z^{-1}+a_{2} z^{-2}+a_{3} z^{-3}}
\end{aligned}
$$

\section{B. Dynamics of Four Quadrant Detector and Laser Beam}

A four quadrant photo sensitive detector (PSD) has four photosensing parts arranged in four quadrants, respectively. Usually, the laser beam is pointed towards the dead center between the 4 quadrants and the beam diameter is chosen to fit within the PSD area. When light falls on all PSD quadrants, they generate currents for each quadrant according to the light intensity and then amplified into voltage signals $V_{1}$ to $V_{4}$. The difference between the left and right quadrants $\left(V_{x}\right)$ and top and bottom quadrants $\left(V_{y}\right)$ can be used to indicate the offsets of the spot and be adjusted to zero by centering the beam, whereas the sum quadrants voltages corresponding at intensity laser beam $\left(V_{s}\right)$ is at a maximum, that is:

$$
\begin{aligned}
& V_{x}=\left(V_{1}+V_{4}\right)-\left(V_{2}+V_{3}\right) \\
& V_{y}=\left(V_{1}+V_{2}\right)-\left(V_{3}+V_{4}\right) \\
& V_{s}=V_{1}+V_{2}+V_{3}+V_{4} .
\end{aligned}
$$

Fig.4 illustrates the voltage signal $V_{x}\left(V_{y}\right.$ is similar as $\left.V_{x}\right)$ and $V_{s}$. Thus $V_{x}$ and $V_{y}$ channel outputs are directly related to the energy of the laser beam that falls in each quadrant. It is assume that the light intensity on the laser's beam cross section obeys Gaussian distribution. The current generated 


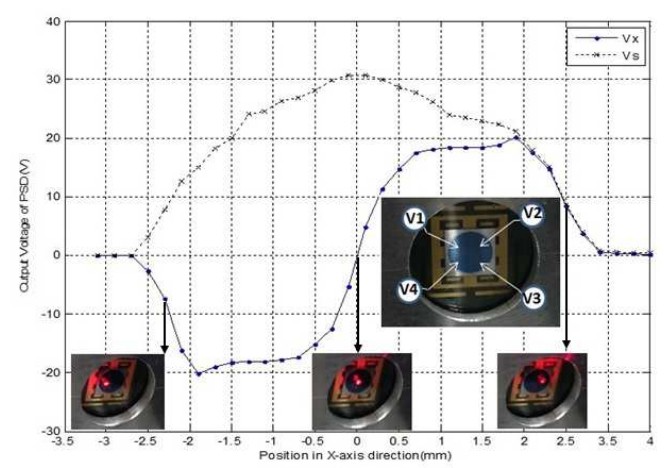

Fig. 4. Output voltage curve $V_{x}$ and $V_{s}$ of the four-quadrant PSD.

by each sensing element can be described as given in:

$$
I=k_{1} \iint \frac{2 E_{l}}{\pi^{2} r} e^{\frac{2\left(x_{1}^{2}+y_{1}^{2}\right)}{r^{2}}} d x_{1} d y_{1}
$$

where $I$ is the current, $r$ the radius of the laser light spot, $E_{t}$ is the energy of the laser beam, $\left(x_{1}, y_{1}\right)$ is the coordinate of a point on the light spot in a coordinates system located at the center of the light spot, and $k_{1}$ is a coefficient. Furthermore, the AFM probe can be used to measure changes in the beam intensity, and used to correct the $V_{x}$ and $V_{y}$ output values for voltage changes due to intensity fluctuations rather than actual beam deviations by filtering.

Moreover, the laser beam motion is assumed similar to the Brownian motion of a particle subjected to excitation and frictional forces. The Brownian motion is given by the generalized differential equation:

$$
\frac{d^{2} x(t)}{d t^{2}}+\beta_{x} \frac{d x(t)}{d t}=W_{x}
$$

were $\beta_{x}$ coefficient of friction and $W_{x}$ is zero-mean Gaussian random variable with variance $\delta_{x}^{2}$. The $y$-axis can be modeled in the same manner as the $x$-axis, though with different dynamics. The discrete state space of (4) laser beam is given by:

$$
\begin{gathered}
X_{k}=A X_{k-1}+B W_{k} \\
Y_{k}=C X_{k-1}
\end{gathered}
$$

with

$$
\begin{gathered}
X_{k}=\left[\begin{array}{llll}
x_{k} & y_{k} & \dot{x}_{k} & \dot{y}_{k}
\end{array}\right]^{T}, A=\left[\begin{array}{cccc}
1 & 0 & \Delta T & 0 \\
0 & 1 & 0 & \Delta T \\
0 & 0 & a_{x} & 0 \\
0 & 0 & 0 & a_{y}
\end{array}\right], \\
B=\left[\begin{array}{cccc}
0 & 0 & b_{x} & 0 \\
0 & 0 & 0 & b_{y}
\end{array}\right]^{T}, C=\left[\begin{array}{cccc}
1 & 0 & 0 & 0 \\
0 & 1 & 0 & 0
\end{array}\right]
\end{gathered}
$$

$\left(x_{k}, y_{k}\right)$ and $\left(\dot{x}_{k}, \dot{y}_{k}\right)$ are the source potion in the plane $x-y$ and velocity respectively.

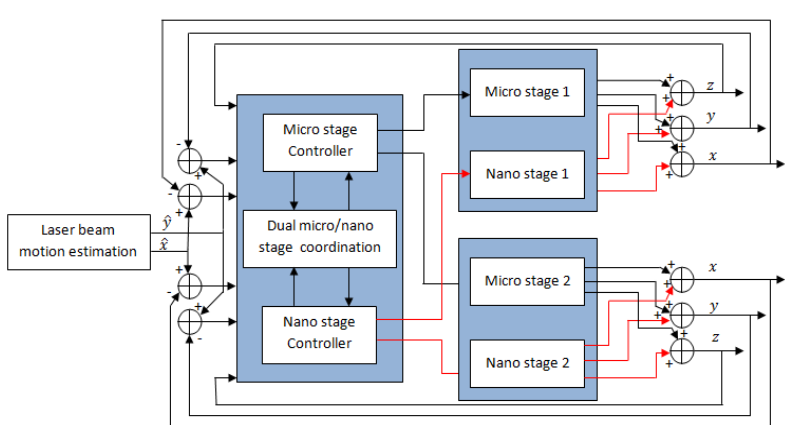

Fig. 5. Master-slaver controller with decoupling structure for maximum light tracking.

\section{Control Scheme of A Two-Fingered} NANOMAnipulation System Tracking a LASER Spot

The analysis of the robotic tasks demonstrates that the handling task, realized by the two-fingered nanogripper, necessitates that the maximum beam intensity should be focused on the microsample in a stable and robust way during several minutes without releasing it. It requires a robust control motion controller of the dual micro/nano manipulators coupled to a laser beam tracking controller to compensate localization uncertainties of laser beam motion. Fig.5 presents the block diagram of the dual-stage controller using the proposed decoupled control structure. A stochastic filter is used to detect and estimate the maximum laser beam intensity in order to anticipate the intensity variations.

\section{A. Particle filter approach}

The first step is to guaranty a real time estimation of the maximum beam intensity during motion. For that, we use a Particle Filer(PF). It approximates the real distribution by generating a set $N$ samples $x_{k}^{i}$ distributed according to the posterior distribution, $p\left(x^{t} \mid y^{t}\right)$, and they associate a normalized importance weight. The adaptive multinominal resampling [14]., based on bootstrap method [15], is used to estimate and predicate the laser beam positions. The corresponding PF approach is presented in Algorithm 1.

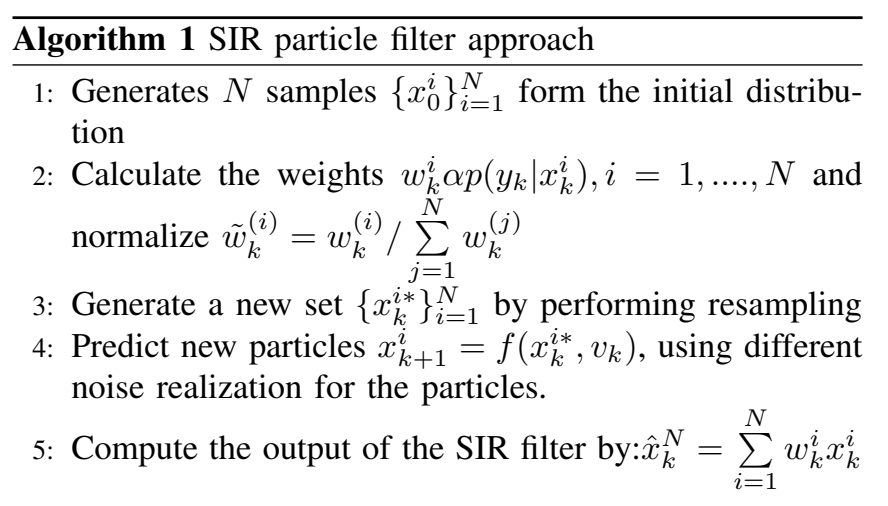

\section{B. $H_{\infty}$ Controller}

The MIMO $H_{\infty}$ standard controller is adopted here in order to provide robustness performances against model 
uncertainties(modeling errors, sensor limitations, nonlinear effects) and environment noises [16] of the piezoelectric actuated nanomanipulators. The magnetic actuated micromanipulators are controlled using a linear PID controller. The Fig. 6 shows the $H_{\infty}$ block diagram where $W_{i}$ represents the weighting function. The $H_{\infty}$ standard problem consists to find a controller $K$ which stabilizes the system and determine a positive number $\gamma_{0}$ to satisfy the following condition:

$$
\|T(s)\|_{\infty}<\gamma\left(\gamma \geq \gamma_{0}\right)
$$

where $T(s)$ represents the closed-loop transfer function of the system represented in Fig.6, given by:

$$
T_{z w}=\left(\begin{array}{ll}
\mathbf{W}_{1} \mathbf{S} & \mathbf{W}_{1} \mathbf{S G} \mathbf{W}_{3} \\
\mathbf{W}_{2} \mathbf{K S} & \mathbf{W}_{2} \mathbf{K S G W}_{3}
\end{array}\right)
$$

$\mathbf{S}=(\mathbb{I}+\mathbf{G}(s) \mathbf{K}(s))^{-1}$ is the sensitivity function. To solve the $H_{\infty}$ standard problem, we need the appropriate choice of the structure weighting functions with optimal parameters which reflect the robust stability and performance requirements. Considering that the axis coupling is negligible, lets us to choose:

$$
\mathbf{W}_{i}=\operatorname{diag}\left(w_{i 1}, w_{i 2}, w_{i 3}\right), \text { with } i=1: 3
$$

and

$$
w_{1 i}=\frac{a_{1 i} p+a_{2 i}}{p+b_{1 i}} \quad w_{3 i}=\frac{p+a_{3 i}}{b_{2 i} p+b_{3 i}} \quad w_{2 i}=\text { const }_{i}
$$

The inverse of $w_{1 i}$ and $w_{3 i}$ are an upper bound on the desired sensitivity loop shape and complementary sensitivity function $T(s)$, the inverse $w_{2 i}$ will effectively limits the controller $\mathbf{u}[17]$.

\section{Parameters optimization}

The GA approach[18] is used to compute the parameter weighting functions namely: $a_{1 i}, a_{2 i}, a_{3 i}, b_{1 i}, b_{2 i}, b_{3 i}$ and const $_{i}$, and to obtain an optimal $H_{\infty}$ controller. The concept is to generate a population of chromosomes representing the parameters to optimize, and subjected to check whether it satisfies the performance index or not. If any the chromosomes does not satisfy the performance index a new chromosome is generated repeatedly until satisfied. The controller synthesis is realized with the following computing procedure form (see Algorithm 2):

For displacement on $x$-direction, the computing procedure allows to obtain the following weighting function:

$$
\mathbf{W}_{11}=\frac{s+1}{s+0.9258} \quad \mathbf{W}_{31}=\frac{s+0.005935}{0.9613 s+0.9032} \quad \mathbf{W}_{21}=0.7097
$$

The optimized discrete transfer function $\mathbf{K}(z)$ is given by:

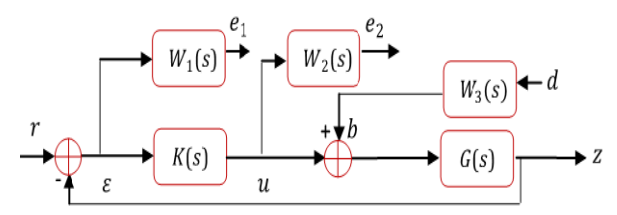

Fig. 6. $H_{\infty}$ structure

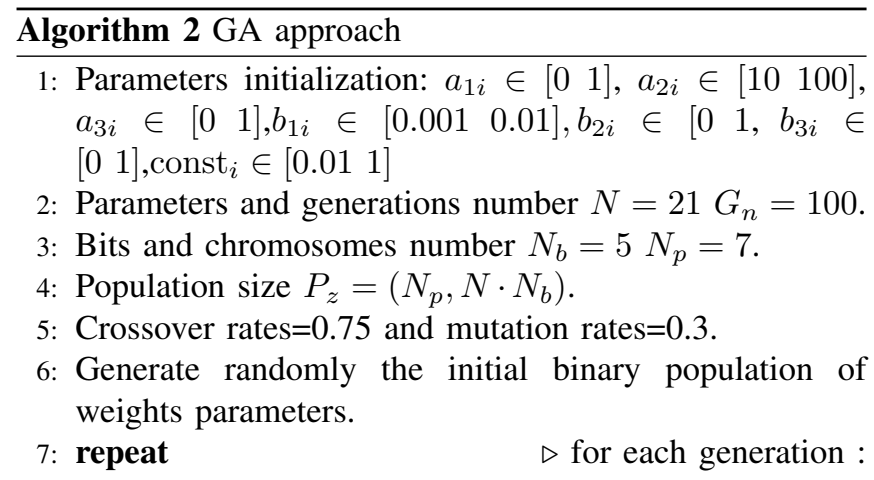

8: Convert binary population to a decimal population:

$$
q(r)=[\max (r)-\min (r)] / 2^{N-1}, \quad 1 \leq r \leq N
$$

9: Evaluate the individual fitness function of all individuals: for $i \leftarrow 1, N_{p}$ do $f_{i}=\|T(s)\|_{\infty}$ end for

10: Apply the Elitism selection technique to select the best-fit individuals for reproduction

11: $\quad$ compute the means fitness: $F=\sum_{i=1}^{N_{p}} f_{i}, F_{m}=\frac{f_{i}}{F}$

12: for each chromosome, compute:

13: for $j \leftarrow 1, N_{p}$ do $e_{j}=\frac{f_{j}}{F_{m}}$ end for

14: (where $f_{i}=$ array of fitness function of chromosomes) the chromosome $\mathrm{i}$ is reproduced integer part of $e_{j}$

15: Breed new individuals through crossover and aleatory mutation operations of genes to obtain new chromosomes

16: Replace least-fit population with new individuals

17: After obtain optimal weights, use Riccati approach to synthesize $H_{\infty}$ controller and use a reduction method based on singular values to reduce the controller order. 18: until last generation

$$
\mathbf{K}_{x}(z)=\frac{1.186 z^{4}-4.01 z^{3}+5.623 z^{2}-3.953 z+1.154}{z^{4}-3.25 z^{3}+4.405 z^{2}-3.06 z+0.9044}
$$

then, we obtain $u_{x}(k)$.

Fig.7 represents the fitness evolution for each chromosome of the population by generation. As one can see, Fig.7(a) demonstrates a rapid convergence of the fitness. For each generation is selected the chromosome population having a maximum fitness as shown in Fig.7(b). Once again, a rapid convergence of the fitness parameter to an optimal value $\gamma_{o p t}=1.01$ is observed, which assumes that many solutions of parameters are envisaged. As GA exhibits an excellent characteristic of global search and selection technique, the chromosomes converge to the same optimal value. Fig.8 represents the $\mathbf{W}_{31}$ parameters optimization evolution and illustrates the parameters optimization convergence.

\section{EXPERIMENTAL RESULTS}

\section{A. Particle filter validation}

To evaluate the performances of the proposed PF, used to estimate the laser beam position, we controlled the laser beam trajectory following a synthetic trajectory generated randomly without working zone. For particle filtering, the particles number is determined such as the computational remained suitable for real-time applications. After several 


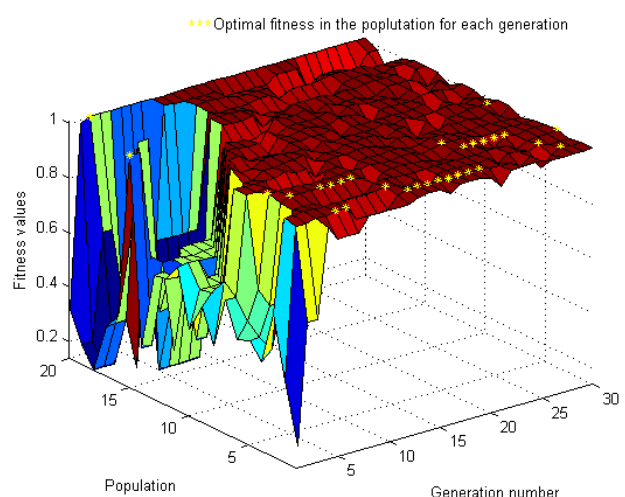

(a)

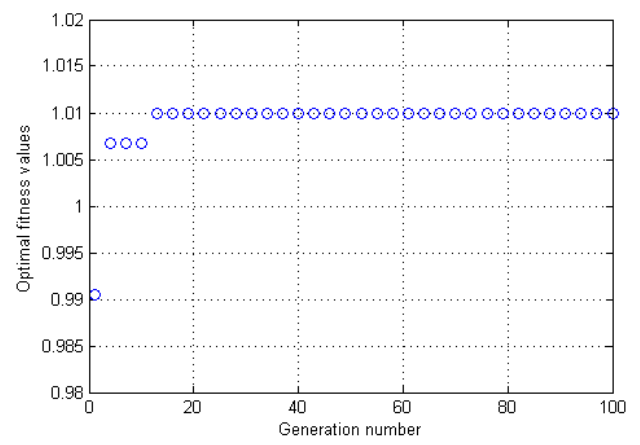

(b)

Fig. 7. Fitness evolution: (a): Population fitness computed for each generation, (b): Optimal fitness

trials, the appropriate number of particles used in the estimation step is determined and fixed to $N=80$.

The results of the laser beam motion prediction using the PF are presented in Fig.9. At first glance, the filter succeed to follow the true trajectories very closely. Performance tests were realized by comparing the performances achieved by the PF with a classical Kalman filter. The evolution of the mean quadratic error $\varepsilon(n)$ is represented in Fig.10. The results exhibit that the performances of the PF are better than the classical Kalman filter in terms of position precision. Once again, Fig.11 illustrates that the PF commits less error on the estimation of the beam velocity.

\section{B. Nanomanipulation Validation}

The control strategy presented in section IV has been implemented in the experimental platform described in Section II. Fig. 12 shows the microhandling task of a microsphere with a diameter of $20 \mu \mathrm{m}$ in the focus on the laser beam. First, we positioned the laser beam in the sensed region of the four-quadrant PSD sensor. Once the localization and the position estimation of the laser beam intensity sensed by the PSD, the particle filter allowed a maximum local search of the beam intensity reflected on the microshpere and projected on the PSD surface. Fig.12(a) shows the detection of one AFM tip by the laser beam, and illustrates the robustness of the PF to determine the localization of the maximum intensity. Then, the gripping and the transport of the sample

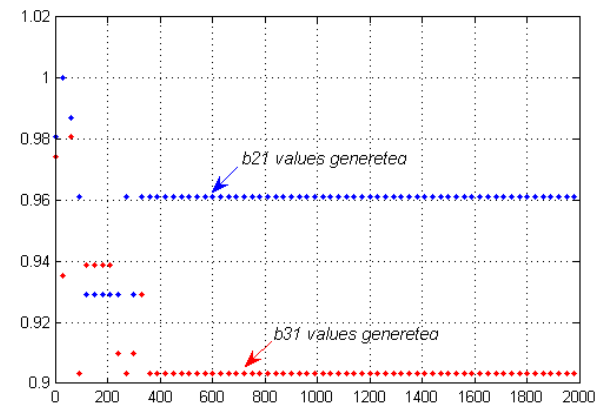

(a)

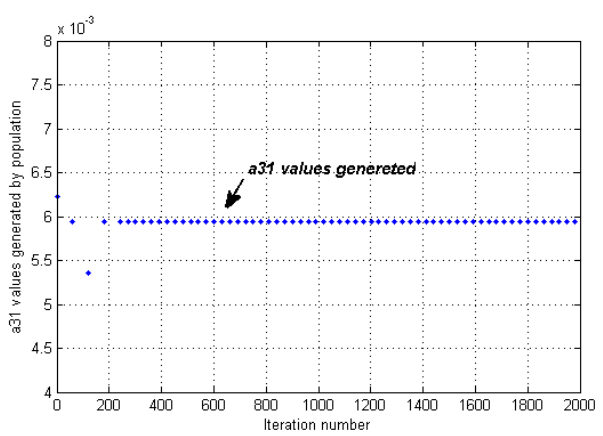

(b)

Fig. 8. $w_{31}$ parameters evolution for $\mathbf{x}$ axis displacement

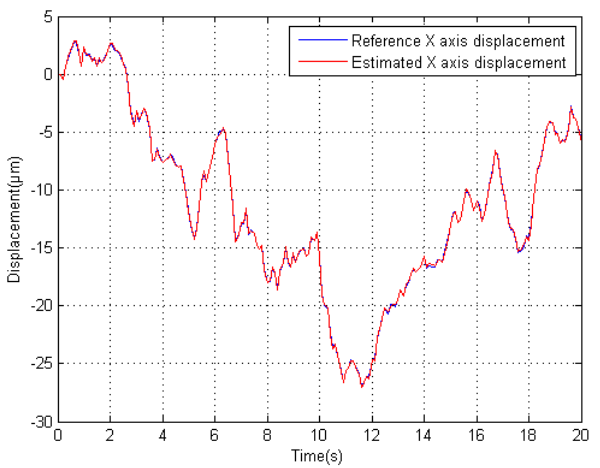

(a)

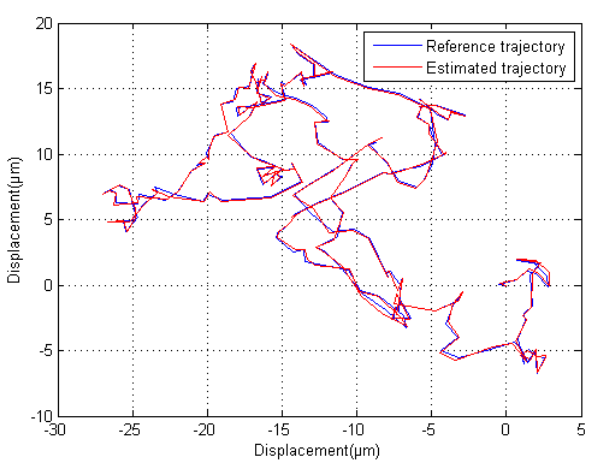

(b)

Fig. 9. Position estimation with particle filter: (a) motion estimation on $x$-direction, and (b) 2D motion 


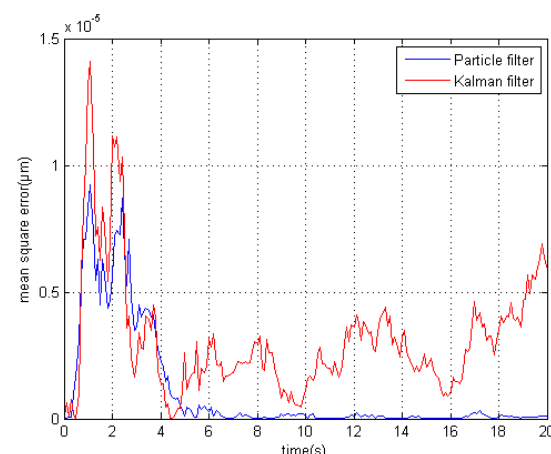

Fig. 10. Mean quadratic position error

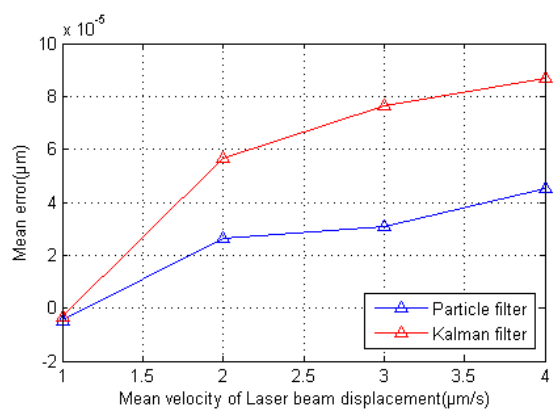

Fig. 11. Mean velocity error

is realized successfully by approaching each tip close to the microsphere (see Fig.12(b)). Finally, the AFM tips are controlled in a cooperative way to handle firmly and robustly the microsphere (cf. Fig.12(c)) before transportation. During transportation, the laser beam tracks efficiently the object until to stabilize its position.

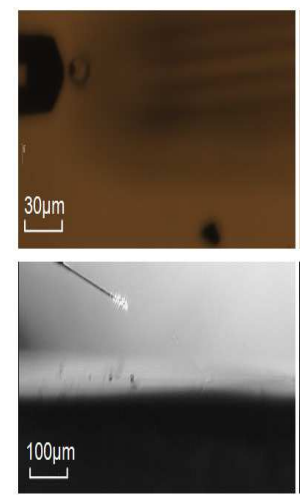

(a)

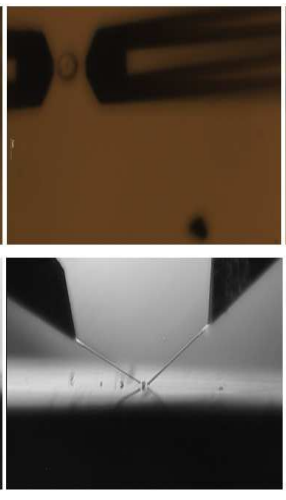

(b)
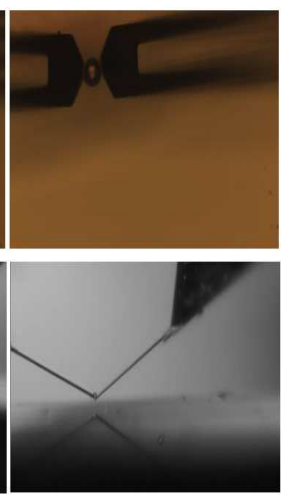

(c)

Fig. 12. Microhandling task of a $20 \mu \mathrm{m}$ spherical bead using a dualnanomanipulation system: (a) detection of the AFM tip\#1 by the laser beam, (b) approach of the AFM tip\#1 and AFM tip\#2 close to the microobject and (c) stable and reliable handling of the microobject.

\section{CONCLUSIONS}

The work presented in this paper deals with the focalization problem of a microsample in the laser beam for radiation applications. The proposed control methodology is based on a two-fingered AFM-based nanogripper handling a microsample illuminated by a laser beam. By processing the maximum beam intensity sensed by a four-quadrant photodiode, the position of the laser beam is detected and located. The main idea of the controller is to guide and to track the beam reflected on the PSD sensor with the cooperative control commands sent to the hybrid micro/nanomanipulators. The experimental results demonstrate the tracking performances when the incident laser beam spot is subjected to external disturbances such as random noises. We used stochastic filters (particle filter and Kalman filter) to localize the laser beam in a three-dimensional space. The preliminary results show that the stability of the microhandling task is preserved during the laser tracking operation.

\section{REFERENCES}

[1] B. Stuhrmann, M. Ggler, T. Betz, A. Ehrlicher, D. Koch, and J. Ks, Automated tracking and laser micromanipulation of motile cells, Review of Scientific Instruments Vol.76, 2005.

[2] Yaakov K.Nahmias and David J.Odde, Analysis of Radiation Forces in Laser Trapping and Laser-Guided Direct Writing Applications, IEEE/Journal of Quantum Electonics, vol.38, No.2, 2002.

[3] M. Sitti, Atomic Force Microscope Probe Based Controlled Pushing for Nanotribological Characterization. IEEE/ASME Transactions on Mechatronics Vo19, N2, 2004.

[4] M. Gauthier, E. Piat, Control of a particular micro-macro positioning system applied to cell micromanipulation. IEEE/Transactions on Automation Science and Engineering Vol.3, N3, 2006.

[5] C. Clévy, A. Hubert, J. Agnus, N. Chaillet, A micro-manipulation cell including a tool changer. Journal of Micromechanics and Microenginneering.15, S292-S301, 2005.

[6] Zhaolong Shen and B.Andersson, Tracking Nanometer-Scale Fluorescent Particles in Two Dimensions With a Confocal Microscope, 2009 IEEE/Transactions on Control System Technology, Vol.19, No.5, 2011.

[7] Yanhua Wu and Dong Sun, Stabilizing a flexible beam handled by two manipulators via PD feedback, IEEE/Transactions on Automatric Control, vol45, no,11,pp2519-2164, 2000.

[8] Nestor O.Perez-Arancibia and James S.Gibson, Observer-Based Intensity-Feedbck Control for Laser Beam Pointing and Tracking , IEEE/ Transactions On Control Systems Technology, vol.20, No.1, 2012.

[9] F. Iwata, Y. Mizuguchi, H.Ko and T. Ushiki, Nanomanipulation of biological samples using a compact atomic force microscope under scanning electron microscope observation, Journal of Electron Microscopy Vol.60, 2011.

[10] W. Driesen, T. Varidel, S. Régnier, J. M.Breguet: Micromanipulation by adhesion with two collaborating mobile micro robots. J. Micromech. Microeng. Vol.15, 2005.

[11] T.Won Seo, D.Soo Kang, H.Soo Kim and J.Kim, Dual Servo Control of a High-Tilt 3-DOF Microparallel Positioning Platform, IEEE/Transactions On Mechatronics, vol.14, No.5, 2009.

[12] K.W.Chan, W.H.LiO and I.Y.Shen, Precision Positiong of Hard Disk Drives using Piezoelectric Actuators with Passive Damping, IEEE/Transaction on Mecatronics vol.13, No.1, 2008.

[13] A. Menciassi, A. Eisinberg, I. Izzo, P. Dario, From macro to micro manipulation: models and experiments. IEEE/Transactions on Mechatronics Vol9, N2, 2004.

[14] Rudolph van der Merwe, Nando de Freitas, Arnaud Doucet and Eric Wan, The Unscented Particle Filter, Cambridge University, 2000

[15] B. Efron and R. J. Tibshirani, An Introduction to the Bootstrap, Chapman Hall, 1993.

[16] Irfan Ahmad,Alina Voda and Gilfas Besanon, $H_{\infty}$ Controller Design for High-Preformance Scanning Tunneling Microscope, 2009 IEEE/Conference on Decision and Control and 28th chines Control Conference.

[17] Skogestad, S and Postlethwaite, Multivariable feedback control :analysis and design, John Wiley and Sons, 1996

[18] Haupt, L.Randy, Practical genetic algorithms.2nd ed, John Wiley Sons, 2004. 